\title{
Children with Both Asthma and Depression Are at Risk for Heightened Inflammation
}

\author{
Lilly Shanahan, $\mathrm{PhD}^{1}$, William E. Copeland, $\mathrm{PhD}^{2}$, Carol M. Worthman, $\mathrm{PhD}^{3}$, Adrian \\ Angold, MRCPsych ${ }^{2}$, and E. Jane Costello, $\mathrm{PhD}^{2}$ \\ ${ }^{1}$ Department of Psychology, University of North Carolina at Chapel Hill, Chapel Hill, NC \\ ${ }^{2}$ Developmental Epidemiology Program, Duke University Medical Center, Durham, NC \\ ${ }^{3}$ Department of Anthropology, Emory University, Atlanta, GA
}

\begin{abstract}
Objective-To test whether children and adolescents with co-occurring asthma and depression are at risk for elevated inflammation-concurrently and at the next assessment.

Study design-Up to 6 yearly assessments per person from the prospective, population-based Great Smoky Mountains Study ( $=1420)$ were used, covering children in the community aged 10-16 years old. High-sensitivity C-reactive protein (CRP) was assayed from annual bloodspot collections and provided indicators of elevated inflammation at CRP $>1, \mathrm{CRP}>2$, and CRP $>3$ $\mathrm{mg} / \mathrm{L}$. Depression was assessed with the Child and Adolescent Psychiatric Assessment. Asthma was assessed using a form adapted from the Centers for Disease Control and Prevention National Health Interview Survey.
\end{abstract}

Results-Controlling common covariates of CRP, the co-occurrence of asthma and depression predicted heightened CRP - concurrently and at the next assessment. In turn, elevated CRP was relatively stable from one assessment to the next.

Conclusions-The co-occurrence of asthma and depression in childhood poses a risk for substantially elevated inflammation concurrently and over time, which could contribute to pathophysiological processes involved in the development of additional chronic diseases and also to asthma-related morbidity and mortality.

The acute phase protein C-reactive protein (CRP) is a marker of systemic inflammation that is used as a nonspecific indicator for cardiovascular and metabolic disease risk in adults. Early identification of risk for chronic disease is crucial for effective prevention and intervention. Elevated CRP is a promising biomarker for this purpose because it is detectable during childhood, when it is already associated with precursors of chronic disease, including obesity and vascular changes. ${ }^{1-3}$ But what predicts elevated CRP during the early life course beyond body mass index (BMI)? In adults, inflammatory processes are exacerbated when chronic disease with an inflammatory basis co-occurs with depression. ${ }^{4,5}$ And, these exaggerated immune responses may at least partially be responsible for elevated morbidity and mortality rates in adults with comorbid chronic disease and depression. Is a pattern of exacerbated inflammation already observable in children with comorbid physical illness and depression?

Copyright (C) 2013 Mosby Inc.

Reprint requests: Lilly Shanahan, PhD, Department of Psychology, University of North Carolina at Chapel Hill, CB \#3270, Davie Hall, Chapel Hill, NC, 27599-3270. lilly_shanahan@unc.edu.

The authors declare no conflicts of interest. 
In children, inflammatory markers are reliably elevated when inflammatory-based asthma ${ }^{3,6,7}$ co-occurs with chronic or acute stressors, including poverty and life events. ${ }^{8}$ Such stressors also tend to be associated with depression, which itself can change stress physiology and predict increases in low-grade systemic inflammation over time. ${ }^{9,10}$ Notably, no studies of children with asthma have examined whether its co-occurrence with depression accentuates inflammatory processes. Here, we addressed this gap in research and tested whether children who were experiencing both depression and asthma at the same point in time were at risk for exacerbated inflammation-concurrently and at the next assessment, 1 year later.

\section{Methods}

The Great Smoky Mountains Study (GSMS) is a longitudinal study of the development of psychiatric disorder in youth. ${ }^{11,12}$ A representative sample of 3 cohorts, aged 9-13 at intake was recruited from 11 counties in North Carolina and interviewed yearly to age 16. Potential participants were selected from the population of about 12000 eligible children using a household equal probability, accelerated cohort design. All children scoring above a predetermined cut point on a screening questionnaire assessing risk for psychopathology, ${ }^{13}$ plus a 1-in-10 random sample of the rest were recruited. Of all children recruited, $80 \%(\mathrm{~N}=$ 1420) agreed to participate. About $8 \%$ of the area residents are African American. American Indians constituted only about $3 \%$ of the population of the study area, but, because they are an understudied group, were oversampled to constitute $25 \%$ of the study sample. All statistical analyses used a sampling weight inversely proportional to participants' probability of selection; therefore, results were representative of the population from which the sample was drawn and not biased from the oversampling procedure. Annual participation rates ranged between $74 \%$ and $94 \%$.

A parent figure and the child were interviewed separately by trained interviewers. Before the interviews began, parent and child signed informed consent forms approved by the Duke University Medical Center Institutional Review Board. Each parent and child received an honorarium for their participation. Blood spot samples were obtained at the beginning of each in-person assessment, as follows: 2 finger-prick samples (yielding 10 bloodspots total per visit) were applied to standardized collection paper, immediately refrigerated upon drying, and express-shipped (without refrigeration) to the laboratory within 2 weeks of collection. ${ }^{14}$ Samples were then stored at $-28^{\circ} \mathrm{C}$ until they were assayed in duplicate. Blood spot samples are a reliable and feasible medium for field collection of biomarker data, including high-sensitivity-CRP. ${ }^{14,15}$

Our high-sensitivity assay for CRP in whole-blood spots was a biotin-streptavidin-based immunofluorometric system that improved on a previously published method. ${ }^{15}$ Streptavidin A-coated microtiter plates bind a biotinylated capture antibody to CRP, clone C2. A second Europium-labeled antibody then binds to the streptavidin A biotin-C2-CRP complex; fluorescence of the resultant complex is directly proportional to the CRP concentration in each well. Detail regarding our CRP assays, and the calculation of serum equivalents have been previously published. ${ }^{10,14,15}$ The blood spot method has good precision and reliability. ${ }^{10}$

$\mathrm{CRP}<10 \mathrm{mg} / \mathrm{L}$ in the analytic sample ranged from 0.01-9.71 (mean $=0.77, \mathrm{SD}=1.41)$. Fifty-seven cases (1.2\%; all percentages reported are weighted) were in the CRP $>10 \mathrm{mg} / \mathrm{L}$ range. CRP $>3 \mathrm{mg} / \mathrm{L}$ is a useful cutoff for disease risk in adults, but children typically have lower CRP. ${ }^{16}$ Consistent with previous research, ${ }^{3}$ we examined several dichotomous elevated CRP variables $(>1[19.1 \%],>2[11.2 \%],>3[7.8 \%])$. These cutoffs resulted in reasonably-sized groups and are not sample-specific. Given our focus on exacerbated 
inflammation and research indicating that very high CRP is a powerful predictor of later disease risk, ${ }^{17}$ cases with CRP $>10 \mathrm{mg} / \mathrm{L}$ were included. Children with asthma and depression were more likely to have very high CRP $(\mathrm{OR}=4.15,1.88-9.14 ; P<.004$ and $\mathrm{OR}=13.48,3.41-52.83 ; P<.001$, respectively). Thus, excluding these cases would have biased the analytic sample toward excluding the cases of most interest.

Physical health problems were assessed from parents with a survey adapted from the Centers for Disease Control and Prevention National Health Interview Survey Child Supplement (1988). A dichotomous variable indicated whether asthma, respiratory allergies, or hay fever were present in the past year. Additional dichotomous variables indicated the presence of infections (eg, tonsillitis, ear infection, urinary tract infections), other atopic diseases (eg, eczema), other chronic diseases (eg, diabetes, cancer, arthritis, chronic heart disease), and injuries in the past year. An additional variable summed the number of all illnesses experienced during the past year.

Depression was also measured at each assessment, using the Child and Adolescent Psychiatric Assessment, a structured interview. ${ }^{18}$ The time frame for ascertaining the presence of most behaviors was the past 3 months to minimize forgetting and recall biases. A symptom was counted as present if reported by either parent or child. Information about the date of onset, duration, and intensity of each symptom was used to create diagnostic variables consistent with the Diagnostic and Statistical Manual of Mental Disorders, Fourth Edition. ${ }^{19}$ Depressive disorders included major depression, dysthymia, and depressive disorder not otherwise specified. Two-week test-retest reliability of diagnoses is comparable with that of other highly-structured child psychiatric interviews. ${ }^{20}$ For a discussion of the prevalence rate of psychiatric disorders in the GSMS during childhood. ${ }^{12}$

Controls included age, sex, race, and low socioeconomic status ( $\geq 2$ of the following conditions: income below federal poverty level, low parental education, or occupational status). BMI was calculated from weight and height, measured at each assessment. Substance use (ie, current nicotine, alcohol, and illicit drug use) was assessed in the Child and Adolescent Psychiatric Assessment. Medication use in the past 12 months was assessed using the Child and Adolescent Services Assessment. ${ }^{21}$

Asthma was first assessed at Assessment 2 (ages 10+ years); thus, analyses focused on ages $10-16$. During these ages, interviews with the 1420 participants in the longitudinal study resulted in a total of 5231 observations. During 3900 (74.6\%) of these interviews, blood spots were collected. Bloodspots were unavailable when subjects refused or completed interviews by phone. Of 3900 blood-spots obtained, 3854 (97.3\%) were successfully assayed for high-sensitivity-CRP. Of these cases, $\mathrm{N}=3664$ also had data on asthma and BMI. The mean number of CRP samples per person was $3.14(\mathrm{SD}=1.59$, range $=1-6)$. Cases included vs excluded from the analyses did not differ in terms of asthma and depression $(\mathrm{OR}=0.93,0.70-1.24 ; P=.61$ and $\mathrm{OR}=1.21,0.62-2.40 ; P=.57$, respectively), but were more likely to be American Indian and to be younger $(\mathrm{OR}=1.71,1.37-2.14 ; P<$. 001 and $\mathrm{OR}=0.88,0.83-0.93 ; P<.001$, respectively). Thus, there were 1420 participants in the GSMS; between ages 10-16, the repeated interviews resulted in 3664 observations from these participants with complete CRP and health information. When conducting longitudinal analyses requiring the simultaneous use of at least 2 waves of data, we used 2194 observations.

\section{Results}

Weighted logistic regression models were implemented in a generalized estimating equations framework by SAS PROC GENMOD, specifying an exchangeable covariance 
matrix. The robust variance (sandwich type) estimates derived from generalized estimating equations adjust the SEs of the parameter estimates for the stratified design effects and also multiple assessments per subject; thus, allowing us to generalize statistical estimates to the population from which the sample was drawn. In the logistic regressions, CRP was the outcome, and depression and asthma — assessed at the same point in time—-the predictors; their co-occurrence was captured by a multiplicative interaction term.

\section{Cross-Sectional Analyses}

Table I displays bivariate associations between all study variables with elevated CRP. The Figure displays the weighted percentage of elevated CRP in subgroups defined by asthma and depression. Subjects with both asthma and depression at the same assessment had the highest percentage of elevated CRP. Table II shows results from 3 cross-sectional separate multivariate logistic regression models, and reveals that the interaction between asthma and depression was significant in the prediction of CRP $>2$ and CRP $>3$ in the adjusted models.

\section{Longitudinal Analyses}

In lagged longitudinal analyses, we tested whether the co-occurrence of depression and asthma predicted elevated CRP at the next assessment, controlling for previous elevated CRP and other significant covariates of CRP. Stability of elevated CRP was quite high (OR $=6.64,4.34-10.16 ; P<.001 ; \mathrm{OR}=6.94,4.22-11.43 ; P<.001 ;$ and $\mathrm{OR}=9.16,4.64-18.07$; $P<.001$, for CRP $>1, \mathrm{CRP}>2$, and CRP $>3$, respectively). Importantly, despite this high stability, the co-occurrence of depression and asthma predicted increases in the likelihood of meeting the CRP $>2 \mathrm{mg} / \mathrm{L}$ and $\mathrm{CRP}>3 \mathrm{mg} / \mathrm{L}$ cutoffs at the next assessment $(\mathrm{OR}=13.85$, $1.15-166 ; P=.04 ; \mathrm{OR}=19.18,1.49-246 ; P=.02$; respectively).

\section{Ruling out Alternative Explanations}

BMI did not account for the interaction between asthma and depression in the prediction of elevated CRP (nor did a variable indicating obesity). Longitudinal follow-up analyses indicated that the co-occurrence of CRP and asthma did not predict depression over time and that the co-occurrence of CRP and depression did not predict asthma over time; thus, ruling out alternative directions of effects. Additional variables that could account for the interaction between asthma and depression in the prediction of CRP were also tested-as main effects and in interaction with asthma or depression. Other psychiatric illness (anxiety, behavioral disorders), other physical illnesses (eg, infection), stressors (poverty, maltreatment, family dysfunction), the overall number of illnesses, and specific medication groups (eg, psychotropic vs all other prescribed medication) did not account for our findings. Results also did not change substantially when running the analyses without the American Indian subsample.

\section{Emotional-Cognitive vs Physiological/Somatic Depressive Symptoms}

Finally, because some previous research on depression and inflammation focused on 1 symptom domain of depression only, we created 2 dichotomous variables - one indicating $\geq 1$ emotional-cognitive symptoms (depressed/irritable mood, anhedonia, low self-esteem, suicidal ideation, hopelessness), and the other $\geq 1$ physiological/somatic symptoms (weight loss/gain, insomnia/hypersomnia, psychomotor agitation/retardation, fatigue/loss of energy, difficulty concentrating) - and tested interactions between each of these domains and asthma in the prediction of CRP. Controlling for all covariates, including BMI, the interaction between asthma and emotional-cognitive symptoms predicted CRP $>3(\mathrm{OR}=4.73 ; 1.29$ $17.21 ; P<.05)$, but the interaction between asthma and physiological/somatic symptoms was not significant. Indeed, asthma was only significantly associated with CRP $>3$ in 
children with one or more emotional-cognitive symptoms $(\mathrm{OR}=3.07 ; 1.39-6.78 ; P<.01)$, but not in children without such symptoms.

\section{Discussion}

Results revealed that children and adolescents with co-occurring asthma and depression were at increased risk for first reaching elevated levels of inflammation, which, in turn, were quite stable over time. During childhood, such heightened inflammation is already implicated in the pathophysiological processes that contribute to the development of chronic disease, including functional and morphologic vascular changes ${ }^{1-3}$ involved in the development of cardiovascular disease. Elevated inflammation in children with both asthma and depression could also contribute to morbidity and mortality in this group. ${ }^{22}$

How does the distress characteristic of depression "get inside the body" of children with asthma? ${ }^{8}$ First, children with both depression and asthma may be less likely to seek and comply with treatments for asthma. ${ }^{23,24}$ Reduced treatment compliance has been implicated in poorer outcomes for many diseases that co-occur with depression. ${ }^{24}$ Poor treatment compliance could result in poor asthma control, which could further exacerbate inflammatory processes. ${ }^{25}$ Especially the emotional-cognitive symptoms of depressionsadness, irritability, loss of interest and pleasure, feelings of hopelessness, worthlessness, and guilt - appeared to be associated with exacerbated inflammation, perhaps because they result in reduced motivation to comply with asthma treatments; thus, potentiating health risks.

Second, with their shared inflammatory basis, asthma and depression may synergistically contribute to exacerbated proinflammatory processes. ${ }^{23}$ Research on other chronic illnesses with an inflammatory basis also shows exacerbation of inflammation in the presence of depression. Neuroendocrine processes involving glucocorticoid hormones that regulate the body's immune response may, in part, be responsible for these exacerbations. ${ }^{9}$ It is also possible that children with both asthma and depression have exacerbated responses to environmental agents, including pollutants. Third, co-occurring asthma and depression could reduce resistance to infections, which, in turn, could further exacerbate asthma and inflammation. Our analyses adjusted for other illnesses, including infections, and participants were only interviewed when healthy; thus, this explanation is less likely. Finally, although our analyses ruled out a number of alternative explanations, it is possible, that the co-occurrence of asthma and depression is simply a marker of exacerbated inflammation without having a causal role in the exacerbation of inflammatory processes. Many of these proposed mechanisms could be tested in studies of medically verified asthma that measure both: systemic inflammation and also inflammatory markers most relevant to the development and maintenance of asthma.

The GSMS is a community study; therefore, our measure of asthma and all other medical conditions was parent-based and not verified by a physician. Replications of this study should aim for multi-informant measures of health, including adolescents' self-reports. Second, our measure assessed the presence but not the severity of asthma. It is possible that our findings apply only to cases with moderate to high asthma severity. Third, fewer than 30 children had co-occurring depression and asthma. This cell size is consistent with other community samples exploring heterogeneity in the depression-CRP link, which have provided important new directions for research. ${ }^{26}$ Nevertheless, our results should be interpreted with some caution, and replication is important. Fourth, future studies should test the generalizability of our findings to other health outcomes. In adults, a range of diseases (eg, cancer, diabetes, HIV/AIDS, rheumatoid arthritis) have also been linked to worse medical outcomes when comorbid with depression, ${ }^{4,5}$ and exacerbated immune responses 
may at least partially be responsible for these findings. Finally, CRP was the only inflammatory marker available in the GSMS, but our findings should be replicated with other markers of systemic inflammation such as interleukin-6.

We have identified a subgroup of children who may be at particular risk for early and stable exacerbated inflammation. From a population perspective, these findings apply to a significant number of children. Health care providers should be aware that children with cooccurring asthma and depression may be at particular risk for exacerbated inflammation and related health complications, ${ }^{22}$ and that this risk may be highest in youth whose depression is predominantly characterized by emotional-cognitive symptoms. Aggressive treatments and monitoring for compliance with treatments may be particularly important for this subgroup. ${ }^{9}$

\section{Acknowledgments}

Supported by the National Institute of Mental Health (MH63970, MH63671, MH48085, MH094605), the National Institute on Drug Abuse (DA/MH11301), Brain \& Behavior Research Foundation (formerly NARSAD) (Early Career award to W.C.), and the William T. Grant Foundation.

The authors thank the children and their parents for their participation in this study.

\section{Glossary}

BMI Body mass index

CRP C-reactive protein

GSMS Great Smoky Mountains Study

\section{References}

1. Kapiotis S, Holzer G, Schaller G, Haumer M, Widhalm H, Weghuber D, et al. A proinflammatory state is detectable in obese children and is accompanied by functional and morphological vascular changes. Arterioscl Thromb Vasc Biol. 2006; 26:2541-2546. [PubMed: 16973973]

2. Reinehr T, Kiess W, de Sousa G, Stoffel-Wagner B, Wunsch R. Intima media thickness in childhood obesity: Relations to inflammatory marker, glucose metabolism, and blood pressure. Metabolism. 2006; 55:113-118. [PubMed: 16324929]

3. Skinner AC, Steiner MJ, Henderson FW, Perrin EM. Multiple markers of inflammation and weight status: cross-sectional analyses throughout childhood. Pediatrics. 2010; 125:801-819.

4. Carney RM, Freedland KE, Steinmeyer B, Blumenthal JA, Berkman LF, Watkins LL, et al. Depression and five year survival following acute myocardial infarction: a prospective study. J Affect Disord. 2008; 109:133-138. [PubMed: 18191208]

5. Lutgendorf SK, Lamkin DM, DeGeest K, Anderson B, Dao M, McGinn S, et al. Depressed and anxious mood and T-cell cytokine expressing populations in ovarian cancer patients. Brain Behav Immun. 2008; 22:890-900. [PubMed: 18276105]

6. Dodig S, Richter D, Zrinski-Topić R. Inflammatory markers in childhood asthma. Clin Chem Lab Med. 2011; 49:587-599. [PubMed: 21303302]

7. Fujita M, Ueki S, Ito W, Chiba T, Takeda M, Saito N, et al. C-reactive protein levels in the serum of asthmatic patients. Ann Allergy Asthma Immunol. 2007; 99:48-53. [PubMed: 17650829]

8. Chen E, Miller GE. Stress and inflammation in exacerbations of asthma. Brain Behav Immun. 2007; 21:993-999. [PubMed: 17493786]

9. Irwin MR, Miller AH. Depressive disorders and immunity: 20 years of progress and discovery. Brain Behav Immun. 2007; 21:374-383. [PubMed: 17360153] 
10. Copeland WE, Shanahan L, Worthman C, Angold A, Costello EJ. Cumulative depressive episodes predict later C-reactive protein levels: a prospective analysis. Biol Psychiatry. 2012; 71:15-21. [PubMed: 22047718]

11. Costello EJ, Angold A, Burns BJ, Erkanli A, Stangl DK, Tweed DL. The Great Smoky Mountains Study of Youth: functional impairment and serious emotional disturbance. Arch Gen Psychiatry. 1996; 53:1137-1143. [PubMed: 8956680]

12. Costello EJ, Mustillo S, Erkanli A, Keeler G, Angold A. Prevalence and development of psychiatric disorders in childhood and adolescence. Arch Gen Psychiatry. 2003; 60:837-844. [PubMed: 12912767]

13. Achenbach, TM.; Edelbrock, C. Manual for the Child Behavior Checklist and Child Behavior Profile. Burlington, VT: University of Vermont; 1983.

14. Worthman CM, Stallings JF. Hormone measures in finger-prick blood spot samples: New field methods for reproductive endocrinology. Am J Phys Anthropol. 1997; 103:1-21. [PubMed: 9185948]

15. McDade T, Burhop J, Dohnal J. High-sensitivity enzyme immunoassay for C-reactive protein in dried blood spots. Clin Chem. 2004; 50:652-654. [PubMed: 14981035]

16. Cook DG, Mendall MA, Whincup P, Isasi CR. C-reactive protein concentration in children: Relationship to adiposity and other cardiovascular risk factors. Atherosclerosis. 2000; 149:139150. [PubMed: 10704625]

17. Ridker PM, Cook N. Clinical usefulness of very high and very low levels of C-reactive protein across the full range of Framingham Risk Scores. Circulation. 2004; 109:1955-1959. [PubMed: 15051634]

18. Angold A, Costello EJ. The Child and Adolescent Psychiatric Assessment (CAPA). J Am Acad Child Adolesc Psychiatry. 2000; 39:39-48. [PubMed: 10638066]

19. American Psychiatric Association. Diagnostic and Statistical Manual of Mental Disorders Fourth Edition (DSM-IV). Washington, DC: American Psychiatric Press, Inc; 1994.

20. Angold A, Costello EJ. A test-retest reliability study of child-reported psychiatric symptoms and diagnoses using the Child and Adolescent Psychiatric Assessment (CAPA-C). Psychol Med. 1995; 25:755-762. [PubMed: 7480452]

21. Ascher BH, Farmer EMZ, Burns BJ, Angold A. The Child and Adolescent Services Assessment (CASA): description and psychometrics. J Emotional Behav Disord. 1996; 4:12-20.

22. Strunk RC, Mrazek DA, Fuhrmann GS, LaBrecque JF. Physiologic and psychological characteristics associated with deaths due to asthma in childhood. A case-controlled study. JAMA. 1985; 254:1193-1198. [PubMed: 4021061]

23. Bender BG. Risk taking, depression, adherence, and symptom control in adolescents and young adults with asthma. Am J Respir Crit Care Med. 2007; 173:953-957. [PubMed: 16424441]

24. DiMatteo R, Lepper H, Croghan T. Depression is a risk factor for noncompliance with medical treatment: meta-analysis of the effects of anxiety and depression on patient adherence. Arch Intern Med. 2000; 160:2101-2107. [PubMed: 10904452]

25. Walker HA, Chen E. The impact of family asthma management on biology: a longitudinal investigation of youth with asthma. J Behav Med. 2010; 33:326-334. [PubMed: 20373008]

26. Danese A, Moffitt TE, Pariante CM, Ambler A, Poulton R, Caspi A. Elevated inflammation levels in depressed adults with a history of childhood maltreatment. Arch Gen Psychiatry. 2008; 65:409_ 415. [PubMed: 18391129] 
$\because \mathrm{CRP}>1 \backsim \mathrm{CRP}>2 \backsim \mathrm{CRP}>3$

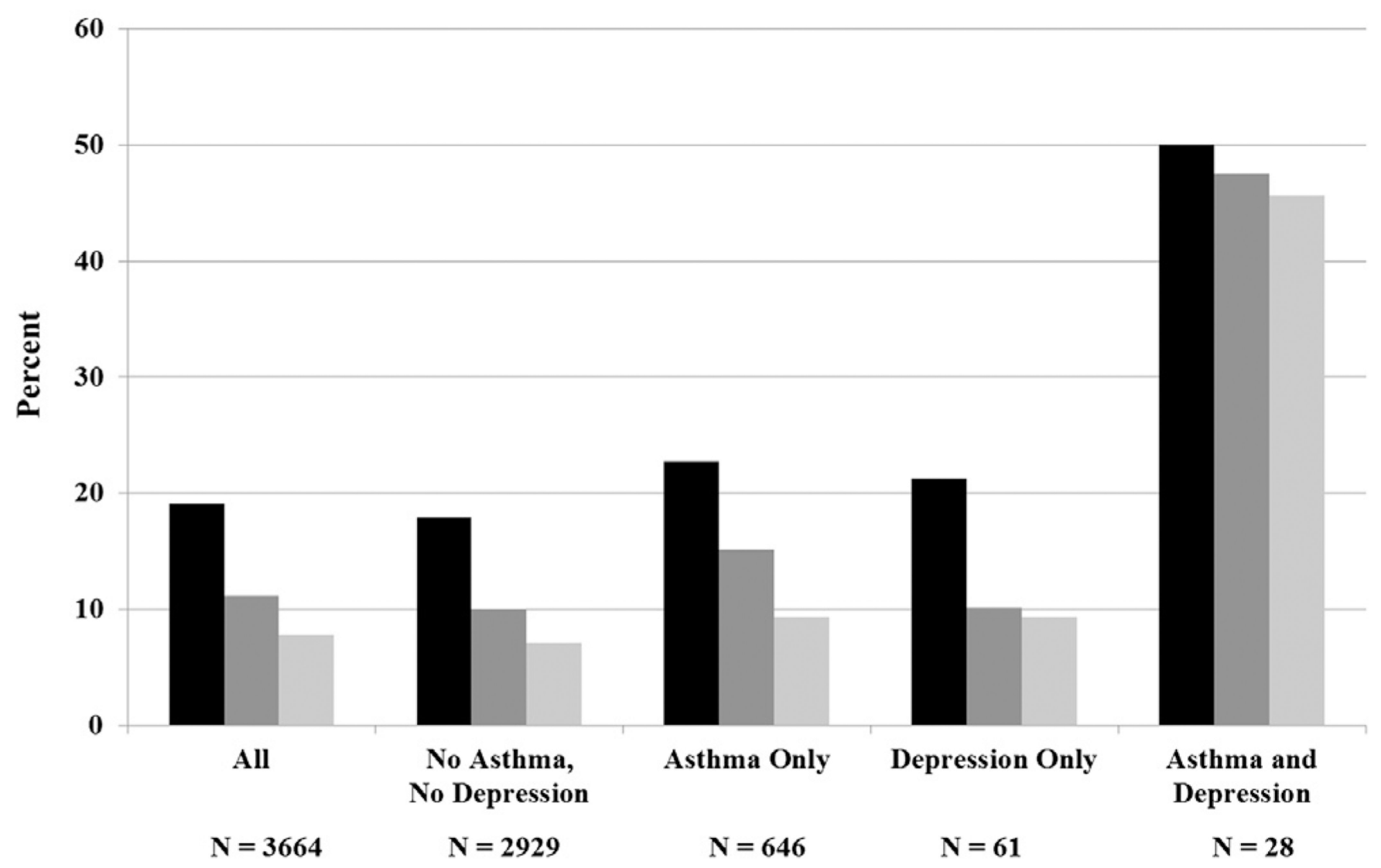

Figure.

Weighted prevalence (\%) of children with elevated CRP for the entire sample and for subgroups defined by asthma and depression. 


\section{Table I}

Bivariate associations with dichotomous elevated CRP variables

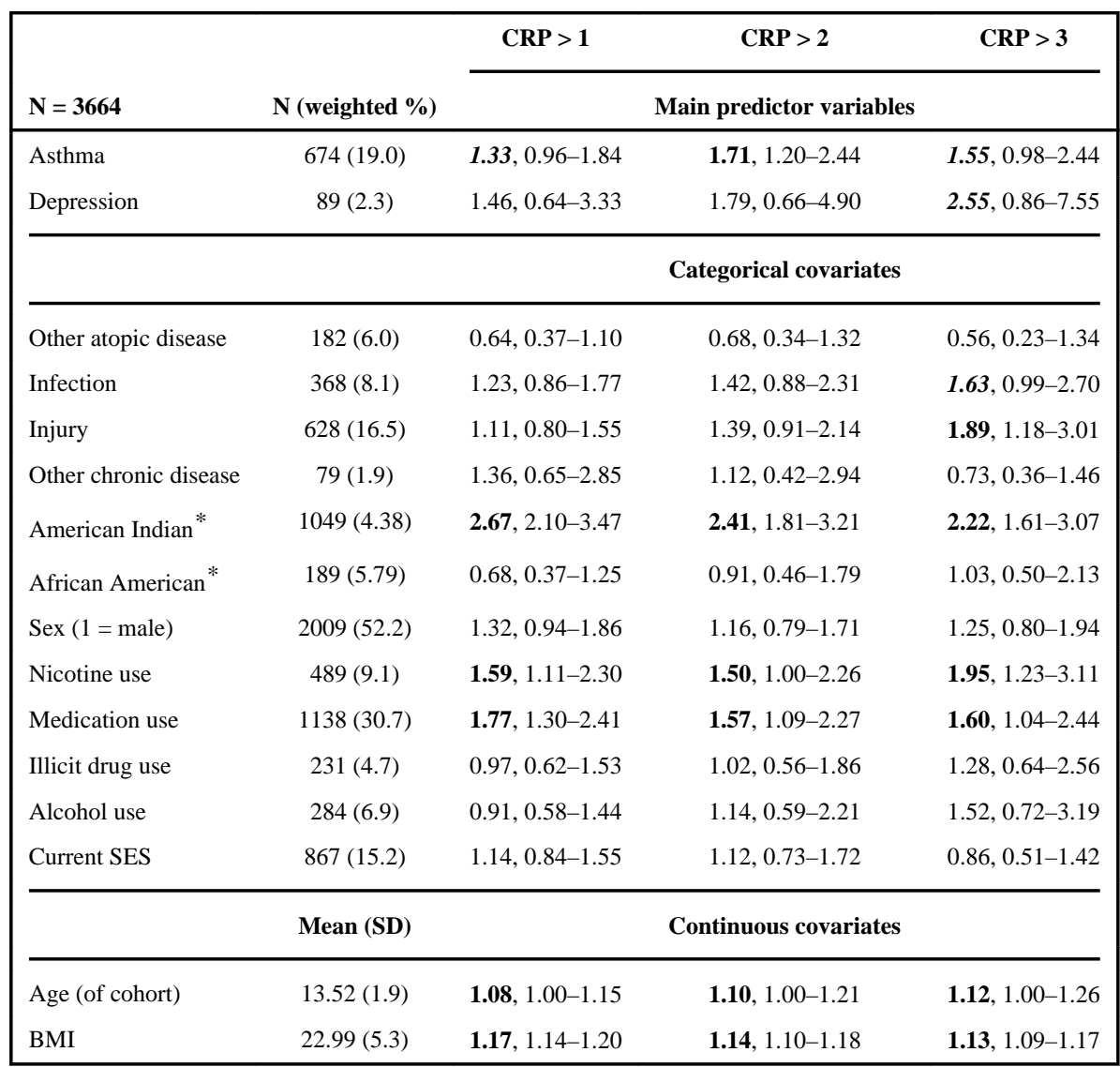

SES, socioeconomic status.

ORs and 95\% CIs were derived from separate weighted logistic regression models for each predictor and each elevated CRP variable. Bolded ORs are significant at $P<.05$. Bolded and italicized ORs approached significance at $P<.10$.

* Reference group $=$ White. 


\section{Table II}

ORs and 95\% CIs derived from 3 separate multivariate weighted logistic regression models predicting each elevated CRP variable, controlling for all covariates

\begin{tabular}{|llll|}
\hline & CRP > 1 & CRP > 2 & CRP > 3 \\
\hline Asthma & $1.06,0.72-1.56$ & $\mathbf{1 . 4 4}, 0.95-2.19$ & $1.21,0.71-2.06$ \\
Depression & $0.82,0.31-2.16$ & $0.63,0.13-3.03$ & $0.72,0.13-4.00$ \\
Depression $\times$ asthma & $\mathbf{3 . 8 3}, 0.83-17.69$ & $\mathbf{7 . 0 2}, 1.13-43.48$ & $\mathbf{1 0 . 0 4}, 1.42-70.96$ \\
\hline
\end{tabular}

Bolded ORs are significant at $P<.05$. Bolded and italicized ORs approached significance at $P<.10$. 